\section{DIDMOAD (Wolfram) syndrome}

SIR: The DIDMOAD (Wolfram) syndrome (Diabetes insipidus, diabetes mellitus, optical atrophy and deafness) is a rare, autosomal, recessively inherited disabling disorder of yet unknown aetiology. Additional neurological symptoms, for example ataxia, dysarthria, nystagmus, dysdiadochokinesia, dysphagia, anosmia, and electroencephalogram (EEG) changes may also be present (Lancet, 1986). Furthermore, psychiatric alterations, such as episodes of severe depression, psychosis, organic brain syndrome, and impulsive verbal and physical aggression, have been documented in about $25 \%$ of these patients; also heterozygote carriers of the gene seem to be predisposed to significant psychiatric illness (Swift et al, 1991).

Case report. We report the case of a 27-year-old female patient with DIDMOAD syndrome. who was in a somnolent state (with normal metabolic and electrolyte profile) and recovered after discontinuation of anticholinergic medication she had received for urge incontinence. Physical examination showed hyposmia, non-reactive pupils in response to light. pale optic discs, diminished gag and uvular reflexes, and diminished tongue motility. In the course of her illness she had developed organic personality syndrome according to DSM-III R (American Psychiatric Association, 1987) with impaired impulse control, affective incontinence, deficient judgement and reduced initiative.

Her computerised axial tomographic (CAT) scan revealed enlarged third and fourth ventricles, slight atrophy of the cerebellum. a small pons and mesencephalon, and fibrous dysplasia of several skull bones. In the EEG a slowed basic rhythm persisted; in the auditory-evoked potentials of the brainstem. there were normal interpeak latencies but diminished V amplitudes. Organ-specific IgG-class autoantibodies against epitopes of central nervous system tissue and against several gangliosides (GM I, GD la, and GT Ib) (Klein e't al, 199l) were detected in plasma: unfortunately, lumbar puncture for the respective antibody tests was refused by the patient. Cytogenetic studies revealed a normal female karyotype (46, XX). Amino acid analysis showed unremarkable normal results; there was no evidence for diminished concentrations of thiamine in serum, erythrocytes, and of intra-erythrocyte thiamine pyrophosphate levels, which have been proposed to cause neuronal degeneration (A. Speitling, personal communication).

While in other patients no signs of abnormality could be found, this is not so for a 32-year-old woman (Kehl \& Keller, 1982); the second report confirming necropsy findings of pontocerebellar atrophy in DIDMOAD syndrome by CAT scan. The possible role of an auto-immune process in the pathogenesis of DIDMOAD syndrome and its connections to the still poorly characterised psychiatric symptoms of this disorder is proposed by our case and warrants further research.
American Psychia tric Association (1987) Diagnostic and Statistical Manual of Mental Disorders (3rd edn, revised) (DSM-III-R). Washington, DC: APA.

LANCET (1986) DIDMOAD (Wolfram) syndrome. Lancet, $i$. $1075-1076$.

KFHL, O. \& KELleR, U. (1982) DIDMOAD-Syndrom (Diabetes mellitus, Diahetes insipidus, optic atrophy, deafness) mit zerebellopontiner atrophie. Schweizer Medizinische Wochenschrift, 112, 348-352.

Klein, R., Richter, C. \& Bi:Rg, P. A. (1991) Antibodies against central nervous system tissue (anti-CNS) detected by ELISA and western blotting: marker antibodies for neuropsychiatric manifestations in connective tissue disease. Journal of Autoimmunity. 10, 133-144.

Swirt, R. G., Pirkins, D. O.. Case, C. L.. el al (1991) Psychiatric disorders in 36 families with Wolfram syndrome. American Journal of Psychiatry, 148, 775-779.

M. Kellner F. Strian

Max-Planck-Institute of Psychiatry

K. FASSBENDER

Kraepelinstrasse 10

80804 Munich. Germany

University of Ulm

I. KENNERKNECHT

Germany

University of Tübingen

R. KLEIN

Germany

\section{Cognitive impairment and clozapine}

SIR: We wish to report the association of a deterioration in memory for verbal and visual material with high doses of clozapine in a patient with schizophrenia.

Case report. The patient was referred to the rehabilitation services following an 18-month in-patient admission. She had spent her early years in Ghana but was educated in the UK and had a good academic record, achieving eight ' $O$ ' levels and one ' $A$ ' level. Her family report that she had been unwell for approximately 16 months before presenting to the psychiatric services, during which time she had returned to Ghana and had an unplanned pregnancy.

During the 18-month admission she developed a systematised delusion that her baby was a chicken, reported hearing the voices of angels talking to her, and believed that her mind was being controlled by an outside force. Clozapine up to a maximum dose of $500 \mathrm{mg}$ daily was used to relieve her psychotic symptoms, and maintained on discharge.

On transfer to rehabilitation services the patient had no psychotic symptoms but reported a three-month history of deterioration in her memory. Specifically, she was now unable to find her way to the nursery with her small child, regularly forgot appointment times, and had difficulty finding offices she had visited several times previously. Mental state examination and neurological examination revealed no abnormality. A computerised tomographic scan was reported as normal, and electroencephalography showed 